\title{
Residual Stress Redistribution due to Removal of Material Layers by Electrolytic Polishing
}

\author{
I. Surtee ${ }^{1, a^{*}}$ and J.P. Nobre ${ }^{b}$ \\ School of Mechanical, Industrial and Aeronautical Engineering, University of the Witwatersrand, \\ Johannesburg, Private Bag 3, WITS 2050, South Africa \\ ${ }^{1}$ Graduate Mechanical Engineer, Degree and concerned research was carried out as a student \\ at the University of the Witwatersrand \\ a isosurtee@gmail.com, bjoao.nobre@wits.ac.za
}

\section{Keywords: Electrolytic Polishing, Residual Stresses, X-Ray Diffraction}

\begin{abstract}
The phenomenon of redistribution of residual stress due to the removal of material layers by electrolytic polishing can be attributed to the disturbance in the initial equilibrium state of the stress caused by the material removal, which must be considered when this technique is used together with X-ray diffraction for residual stress determination. This study investigates these redistributions and involves the development of a numerical simulation model on the ANSYS finite element platform, in which different material removal cases were simulated on a flat plate model. The cases simulated and compared includes the removal of complete layers in incremental depths, the removal of strip layers in incremental widths and depths as well as the removal of square sections in incremental sizes and depths. The main objective deals with the effect of removal depth for the full layer removal and the effect of plate geometry for the square and strip removal models. A mathematical correction technique that was shown to correct the redistributions within minimal limits for the square layer removal process is proposed. Moore and Evans correction as well as Pederson-Hanson correction techniques are currently being used, but both methods are not unique to square section removal corrections and were used for model validation. The correction proposed displayed promising results when applied to simulated data, but lacked enough data and experimental validation to be generalized. The findings show a lot of insight into the redistribution patterns of these stresses as well as the different parameters that affect their behavior.
\end{abstract}

\section{Introduction}

Residual stresses can be defined as the stresses locked in a material, which are in equilibrium in its interior without any external forces acting, due to any heterogeneity of deformation, at micro or macro scale, of different origins, such as mechanical, thermal and metallurgical. The knowledge of the residual stresses and their behavior in a material are of great importance as they affect the performance of the material and ultimately they are often responsible for the failure of mechanical components, or lifetime improvement when skillfully generated [1].

Residual stresses in crystalline materials can be analyzed non-destructively by the X-ray diffraction (XRD) method, measuring the induced lattice deformations using the lattice distance of specific $\{\mathrm{hkl}\}$ planes as gage length. In the presence of a given stress state, the lattice deformation is a function of the orientation of the diffracting $\{\mathrm{hkl}\}$-planes relative to the sample's surface. The orientation-dependent variation of the lattice deformation can be related to the underlying stress state. Assuming a plane stress state (due to the small penetration depth of X-rays in metallic materials), the so-called $\sin ^{2} \psi$ method [2] can be used to determine the corresponding residual stresses at material's surface, using specific X-ray elastic constants. One of the most common techniques for residual stress depth profiling is the use of the X-ray diffraction technique in conjunction with the electrolytic layer removal technique. The electrolytic polishing method of material removal allows successful depth profiling without introducing additional stresses [1]. 
The major concern of residual stress measurements of materials undergoing layer removal is that of redistribution. When material layers are removed, the equilibrium state residual stress profile inherent in the material is disturbed. This causes a distribution of stress as the stresses try to reach a new state of equilibrium. The concept of redistribution, whereby the removed layer disturbs the equilibrium stress state and the stresses redistribution, introduces the need for a correction of the measured stress to depict the stress state more correctly. The correction accounts for these redistributions. Current correction methods include the Moore and Evans theory [3], Pederson and Hansson theory [4] as well as the Savaria-Bridier-Bocher theory [5].

In this work, a model able to simulate the material layer removal process for residual stress profiles was developed. The effect of removal depth on the redistribution of residual stress for a full layer removal, strip layer removal and square layer removal model was investigated. In addition, the effect of specimen geometry on the redistribution of residual stress for the square and strip layer removal models was also investigated. The effect of removal geometry on residual stress redistribution was compared. Based on the observed trends, a correction methodology and attempt to generalize it has been studied and proposed.

\section{Numerical Study}

A numerical study using the finite element method (FEM) was carried out, using ANSYS code, to analyze and discuss the effect of removal depth on the redistribution of residual stress for a full layer removal, strip layer removal and square layer removal model. For the linear elastic and isotropic material, typical elastic constants for carbon steel, i.e., Young modulus of $210 \mathrm{GPa}$ and a Poisson's ratio of 0.3 , were considered. Since the residual stress model is known to be influenced by the plasticity of a material, a bilinear isotropic hardening feature of the material was included. This will ensure additional accuracy if the yield strength is exceeded. For this necessary numerical study, a 2D-dimensional axisymmetric finite element model using 4-node isoparametric elements was developed. To simulate the redistribution of stress due to the layer removal, a quarter model of a 5 $\mathrm{mm}$ thickness square plate was considered. The effect of a total $0.5 \mathrm{~mm}$ removal layer was analysed. The simulation of the layer removal was carried out in $0.1 \mathrm{~mm}$ thickness steps. A total of 5 simulation steps were thus considered for each case. The removal of each layer step was simulated using the so-called "birth and death" ANSYS [6] code features. The in-depth residual stress profiles, simulated by the finite element model, were generated imposing a corresponding temperature gradient to the restrained model, taking into account the elastic constants of the material and its thermal expansion coefficient. For each material layer, the elements at a given depth were subjected to the following in-depth temperature gradient:

$$
\Delta T(z)=\frac{\sigma^{R S}(z)(1-v)}{\alpha E},
$$

where, $\alpha$ is the thermal expansion coefficient, E the Young modulus, $v$ the Poisson coefficient, $\sigma^{\mathrm{RS}}(\mathrm{z})$ the in-depth equi-biaxial residual stress profile to be generated and $\mathrm{z}$ represents the distance to the surface. The FEM model is full constrained and the simulated temperature, given by the equation above, is imposed to each element of the model. In this work a constant temperature gradient equal to $28{ }^{\circ} \mathrm{C}$ was imposed to obtain a uniform stress of $96 \mathrm{MPa}$. After each removal layer, the results were taken considering a path on the edge of the plate, through the plate's center, where the results are of interest, from the top surface to the bottom. The results were extracted along this path to simulate the residual stress profile along the components depth. In this way, the percentage distribution based on different material removal analyses can be compared and evaluated. It should be noted that even though a plastic deformation could have been simulated, the above mentioned method was used because the focus was not on simulating plastic deformations but rather simulating known common residual stress profiles in an attempt to study redistributions that occur. Modelling plastic deformation may also be done as an alternative for a better real life scenario model. 


\section{Results and Discussion}

With reference to the results obtained for the full layer removal simulation shown in figure 1 the effect of removal depth on the residual stress redistribution can be observed. As the layer removal depth increased, the redistribution of the residual stress was more evident and pronounced.The findings regarding the effect of depth, is validated by the published data found in [7]. In the referenced study a shot peened profile was simulated using the ABAQUS software and the layer removal was simulated on the initial resulting profile [7]. A larger degree of redistribution is observed for the $15 \mu \mathrm{m}$ depth compared to the $5 \mu \mathrm{m}$ depth removal. This correlates well with the results obtained in the full layer removal simulation, shown in Figure 1.

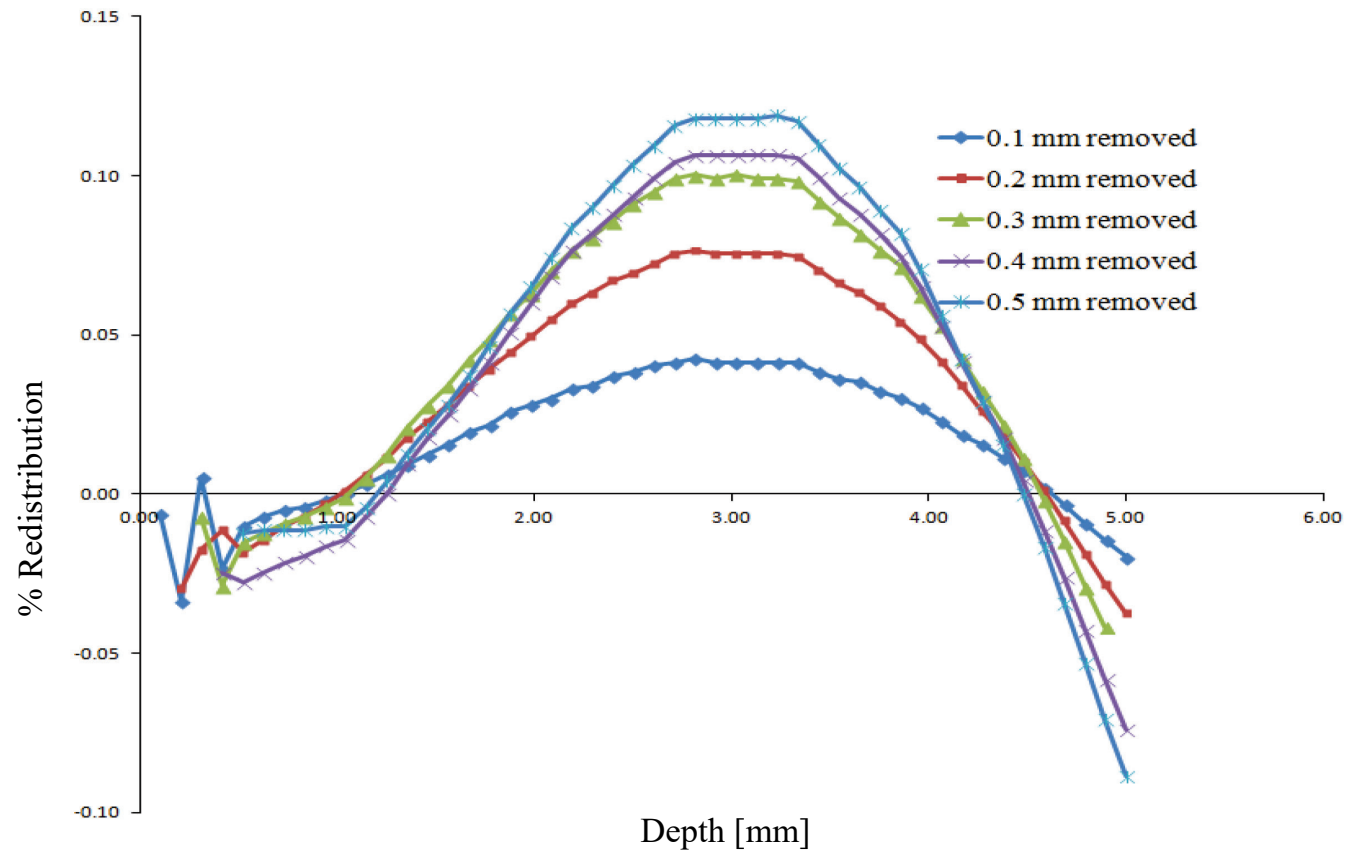

Figure 1- Percentage redistribution of residual stress due to complete layer removal

Considering the Moore and Evans full plate correction, which is a standard correction method for full layer removal, the following Equation 2 below can be written when expanding the integrands using Taylor series and considering that electrolytic polishing deals with small depths of material removal:

$$
\sigma(z 1)=\sigma_{m}(z 1)+\left(-4 \sigma_{m}(H)\left(\frac{\Delta Z 1}{H}\right)\right.
$$

Where $\sigma(z 1)$ is the stress at depth $\mathrm{z} 1, \sigma_{m}(z 1)$ is the measure stress at $\mathrm{z} 1$ and $\mathrm{H}$ is the thivkness of the plate.This equation shows that as removal depth increases $\Delta Z 1$ increases and, hence, the correction required increases, implying that the redistribution increases with the depth. This behaviour, once again, correlates with the results obtained in Figure 1.It was observed that for both the strip removal and square removal results, the redistribution was more pronounced at the surface of the specimen and seems to decrease with depth,shown in figure 2. The Pederson and Hansson study [5] observed a similar redistribution that was more pronounced at the surface for the strip removal simulated, explained it as a notch effect. The notch created by the edge of the removed geometry produces a non-linear distribution and together with the influence of the remaining material adjacent to the groove created, the effect of redistribution is larger at the surface of the material.

The removed geometry is no longer a complete layer but rather a local removal. This implies that a notch effect is created at the edge of the removal geometry creating increased stress gradients in that region, explains the redistribution trend observed. This can be seen in Figure 3. 

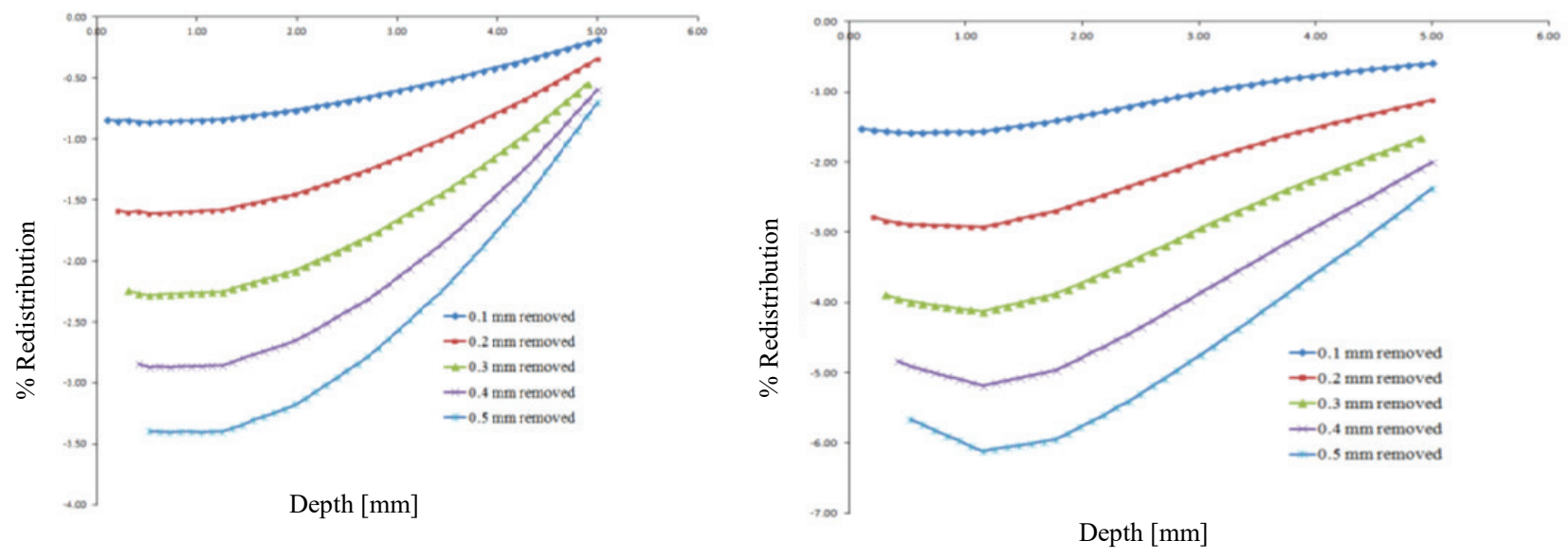

Figure 2-Effect of strip removal (left) and square removal (right).

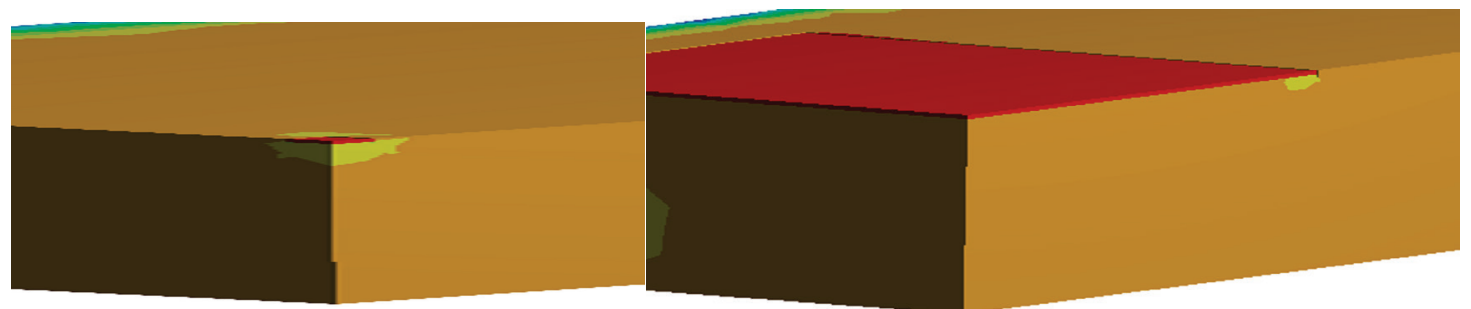

Figure 3-Simulated results depicting the larger effects being experienced at surface.

The explained reasoning regarding the notch effect and the fact that the local removals tend to be larger at the surface and decay with depth, could explain why the change in cross sectional geometry, with constant thickness, results showcased only slight redistribution differences, as shown in Figure 4. In addition, the Moore and Evans correction, although used for full layer removal, did not account for the cross sectional geometry of the specimen, motivating the conclusion that change in cross section for constant thickness does not affect the redistribution. This implies that the redistribution may be independent of the cross sectional geometry of the specimen.
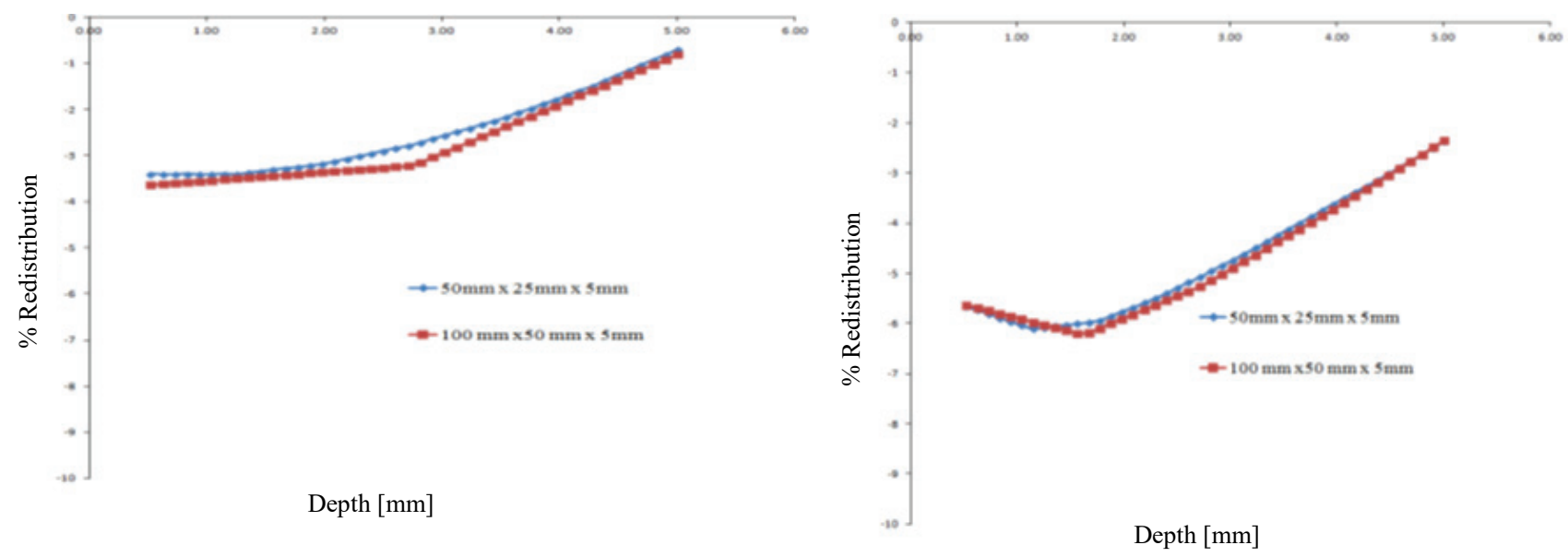

Figure 4-Minimal change in specimens of different cross sections for strip(left) and square(right) removals observed.

Secondly the effect of thickness change for the square and strip removal both depicted that the redistribution is dependent and affected by the thickness of the specimen, as shown in Figure 5. This implies that the redistribution is not independent of thickness of the specimen. The results correlate well with the findings that geometrical changes affect the redistribution of residual stress in a flat plate. In addition, the Moore and Evans correction although used for full layer removal did account 
for original thickness $\mathrm{H}$, motivating the conclusion that change in thickness does affect the redistribution of residual stress during material removal.
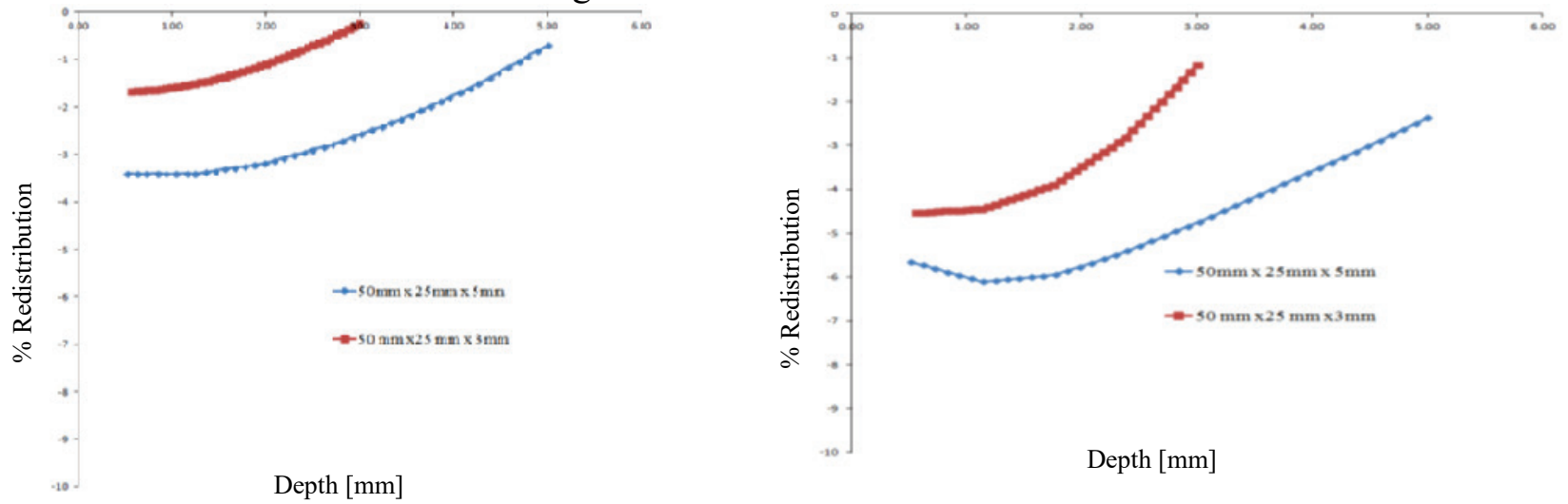

Figure 5-Effect of change in thickness in specimens of same cross section for strip (left) and square (right) removals.

A Correction method was develped for the square removal process. The difference between the full plate value and simulated XRD value was calculated and plotted as a function of depth. A linear regression was carried out for each plotted line and the gradient and intercept values of the lines were noted. The resulting average gradients and intercepts for each model thickness were plotted against corresponding square removal area and resulting trend lines were computed as shown in Figure 6 for a $5 \mathrm{~mm}$ thick plate. The graphs will be used to extract a gradient and intercept, for example for a $1 \mathrm{~mm}$ $\mathrm{x} 1 \mathrm{~mm}$ removal the removal area is $1 \mathrm{~mm}^{\wedge} 2$ hence the extracted gradient is 50.761 and intercept is 6.1536 from the graphs. The correction is then carried out by taking the XRD value + \{extracted gradient (depth) + extracted intercept $\}$ the implemented correction is shown in Figure 7 for a $1 \mathrm{~mm}$ $\mathrm{x} 1 \mathrm{~mm}$ square removal with the extraction equation $50.761(\mathrm{~d})+6.1536$.
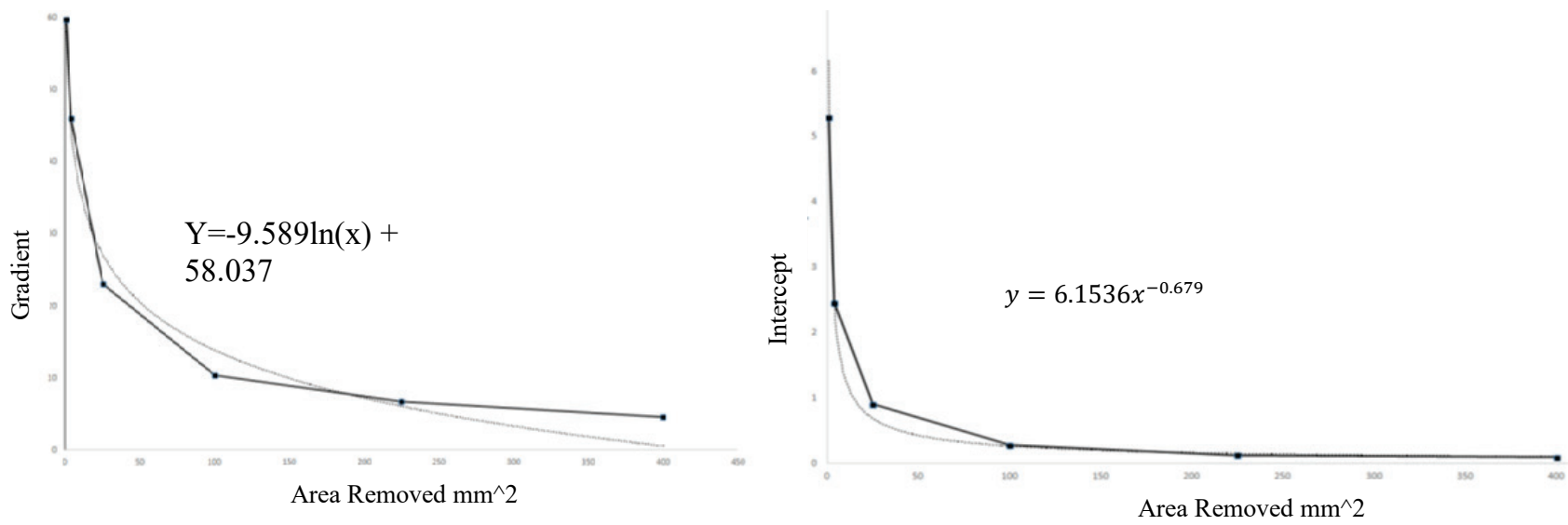

Figure 6 -Correction gradient and intercept extraction graphs proposed for $5 \mathrm{~mm}$ plate with square removal.

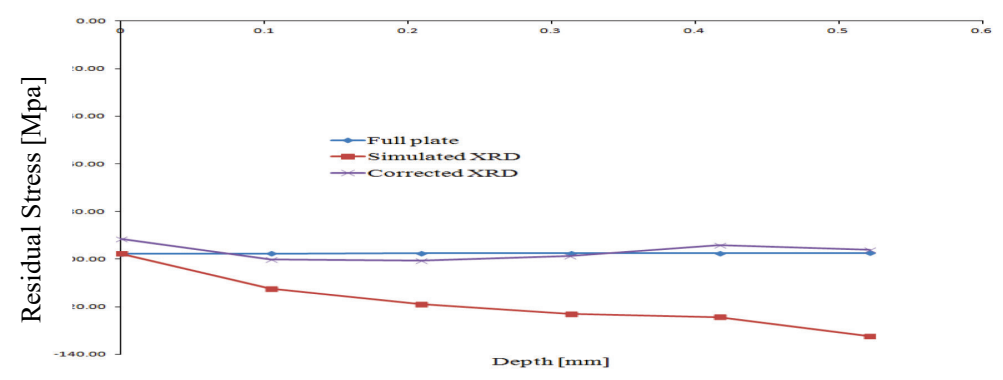

Depth (mm)

Figure 7-Performance of the correction method for a $1 \mathrm{~mm} \times 1 \mathrm{~mm}$ square 
It should however be noted that the simulation and correction was based on the simulated results of two cross sections each and additional work is required to confidently conclude its generality in correction of the data however the correction will serve usefull as the basis for future generalisation attempts. Experimental validation is lacking and experimental procedures should try and evaluate and validate the proposed correction. The correction and all attained results was derived for a constant initial residual stress state and its performance on different stress states such as shot peened samples or samples with bending stress states, is inconclusive.

\section{Conclusions}

1. The percentage redistribution increases as the removal depth increases regardless of removal geometry.

2. The removal geometry of full layer removal, square layer removal and strip layer removal do not cause identical redistribution. The redistribution is dependent on the removal geometry.

3. The geometry of the specimen affects the redistribution observed for square and strip removals; however specimens with constant thickness and different cross sections depicted minimal difference in redistribution. There was not sufficient data to comment on an independence of specimens cross sectional area.

4. The percentage redistribution for the square and strip removal geometries displayed larger distributions at the surface which decreased with depth of the specimen. It was proposed that this was due to high stress gradients caused by the notch and impact of the extra material adjacent to the groove, but this requires experimental validation.

5. The percentage redistribution for the square and strip removal geometries displayed larger distributions for smaller square geometries and smaller widths. The percentage redistribution decreased as the square size or strip width increased. It was proposed that this was due to high stress gradients caused by the notch and impact of the extra material adjacent to the groove being closer to the measurement point at the centroid for smaller squares and strip widths and further away for increased square size and strip widths. This requires experimental validation.

6. A correction methodology was proposed which produced promising results on the tested geometries. It requires experimental validation and possible refinement based on future findings. The correction derivation method itself may prove useful as well.

7. The model itself that was created successfully allows the simulation of the material layer removal process. It also offers a platform for simulating any initial stress state by manipulating the thermal stress feature in the setup.

8. More simulations and experiments are required to validate overall findings.

\section{References}

[1] Handbook of Measurement of Residual Stresses, Society for Experimental Mechanics (SEM), Edited by J. Lu, 1996, pp. 5-34/71-131.

[2] E. Macherauch und P. Müller, Das $\sin ^{2} \psi$-Verfahren der Röntgenographischen Spannungsmessung, Zeitschrift für Angewandte Physik 13 (1961) 305-312.

[3] M. Moore and W. Evans, Mathematical Correction for Stress in Removed Layers in X-Ray Diffraction Residual Stress Analysis, SAE Technical Paper 580035 (1958).

[4] I.L. Hansson and T.F. Pedersen, Finite element calculations for correction of residual stress profiles of coated and uncoated materials measured by X ray diffraction, NDT 22 (1989) 347-352.

[5] V. Savaria, F. Bridier and P. Bocher, Computational quantification and correction of the errors induced by layer removal for subsurface residual stress measurements, Int. J. of Mech. Sci. 64 (2012) 184-195. http://dx.doi.org/10.1016/j.ijmecsci.2012.07.003

[6] ANSYS, 2010, ANSYS Advanced Analysis Techniques, SAS IP, Inc., Houston.

[7] R. F. Laubscher, Gary Styger, Residual stress depth profiling by layer removal, 9th South African Conference on Computational and Applied Mechanics, 2014. 